\title{
Déterminants de la production de plantain dans le sud-ouest du Cameroun : intérêt d'un observatoire
}

\author{
Jean Pierrot ${ }^{a}$, Raphaël Achard ${ }^{b}$, Ludovic Temple ${ }^{c *}$, Catherine Abadie ${ }^{d}$, Roger Fogaine
}

\author{
a Comité d'Expansion \\ économique de l'Allier \\ (CEEA), \\ 12 cours Anatole, \\ 03000 Moulins, France \\ b CARBAP/Cirad-Flhor, \\ BP 832, \\ Douala, Cameroun \\ raphael.achard@cirad.fr \\ c CARBAP, S/c Cirad, \\ BP 2572, Yaoundé, \\ Cameroun \\ I.temple@ camnet.cm \\ d CARBAP/Cirad-Flhor, \\ BP 832, \\ Douala, Cameroun \\ abadie@cirad.fr \\ e CARBAP/Irad \\ BP 832 \\ Douala, Cameroun \\ rfogain7@yahoo.fr
}

* Correspondance et tirés à part

Reçu le 27 mars 2001 Accepté le 22 octobre 2001

Fruits, 2002, vol. 57, p. 75-86 (C) 2002 Cirad/EDP Sciences All rights reserved

DOI: 10.1051/fruits:2002007

Resumen Español, p. 86

\section{Determinant of plantain yields in the southwest of Cameroon: the interest of a production observatory.}

Abstract - Introduction. Plantain is an important crop for food safety in sub-Saharan Africa. In order to treat the research priorities better on a hierarchical basis according to the producer constraints, a permanent observation unit was placed within the framework of an interdisciplinary approach. Materials and methods. A sampling in banana plantations was carried out in the zones of distinct agroecologic contexts which supply Douala (Cameroon). The data were obtained by a follow-up of parcels and holdings. On the parcel scale, observations related to the cultivation methods, the nematode and weevil populations, and the yields. On the holding scale, the follow-ups related to the economic functions of the plantain. Results and discussions. The range of studied farming situations corresponds to a diversity of holding context and objectives. The observations carried out on banana plants (foliar emission rhythm, pseudostem circumference, number of fingers and live leaves, finger weight and length, bunch weight) made it possible to explain how the yield was determined. This is especially affected by the losses before harvest, in relation to the sanitary state of the roots and the nematode and weevil presence. These losses were maximum at the end of the dry season. The yield is also related to the bunch weight determined by the finger number and grade. For this last, the results obtained clearly do not underline the incidence of the black Sigatoka disease. Improvements of the protocol of follow-up are proposed.

Musa (plantains) / Cameroon / surveying / yield components / fungal diseases / parasitism

\section{Déterminants de la production de plantain dans le sud-ouest du Cameroun : intérêt d'un observatoire.}

Résumé - Introduction. Le plantain est une culture importante pour la sécurité alimentaire en Afrique sub-saharienne. Pour mieux hiérarchiser les priorités de recherche en fonction des contraintes des producteurs, une cellule d'observation permanente a été mise en place dans le cadre d'une démarche interdisciplinaire. Matériel et méthodes. Un échantillonnage en bananeraies a couvert les zones de contextes agroécologiques distincts qui approvisionnent Douala. Les données ont été obtenues par un suivi de parcelles et d'exploitations. À l'échelle des parcelles, les observations ont porté sur les pratiques culturales, les populations de nématodes et de charançons, et les rendements. À l'échelle des exploitations, les suivis ont porté sur les fonctions économiques du plantain. Résultats et discussions. La gamme de situations culturales étudiées correspond à une diversité de contextes et d'objectifs des exploitations agricoles. Les observations effectuées sur bananiers (rythme d'émission foliaire, circonférence du pseudotronc, nombre de doigts et de feuilles vivantes, poids et longueur des doigts, poids du régime) ont permis d'expliquer comment se déterminait le rendement. Celui-ci est surtout affecté par les pertes avant récolte, en relation avec l'état sanitaire des racines et la présence de nématodes et de charançons. Ces pertes ont été maximales à la fin de la saison sèche. Le rendement est également lié au poids du régime déterminé par le nombre de doigts essentiellement lié à la croissance et à leur remplissage. Pour ce dernier caractère, les résultats obtenus ne mettent pas clairement en évidence l'incidence de la maladie des raies noires. Des améliorations du protocole de suivi sont proposées.

Musa (plantains) / Cameroun / relevé (des données) / composante de rendement / maladie fongique / parasitisme 


\section{Introduction}

La banane plantain joue un rôle de premier plan dans la sécurité alimentaire des populations d'Afrique sub-saharienne. Si les tonnages commercialisés vers les villes augmentent, les statistiques disponibles indiquent une baisse des disponibilités par habitant [1]. Face à cet enjeu, le Centre africain de recherches sur bananiers et plantains (CARBAP, ex CRBP), localisé au Cameroun, a pour vocation de contribuer au développement durable des productions bananières de consommation locale en Afrique centrale et de l'Ouest. L'un des objectifs est de mettre au point des outils permettant de mieux hiérarchiser les priorités de recherche en

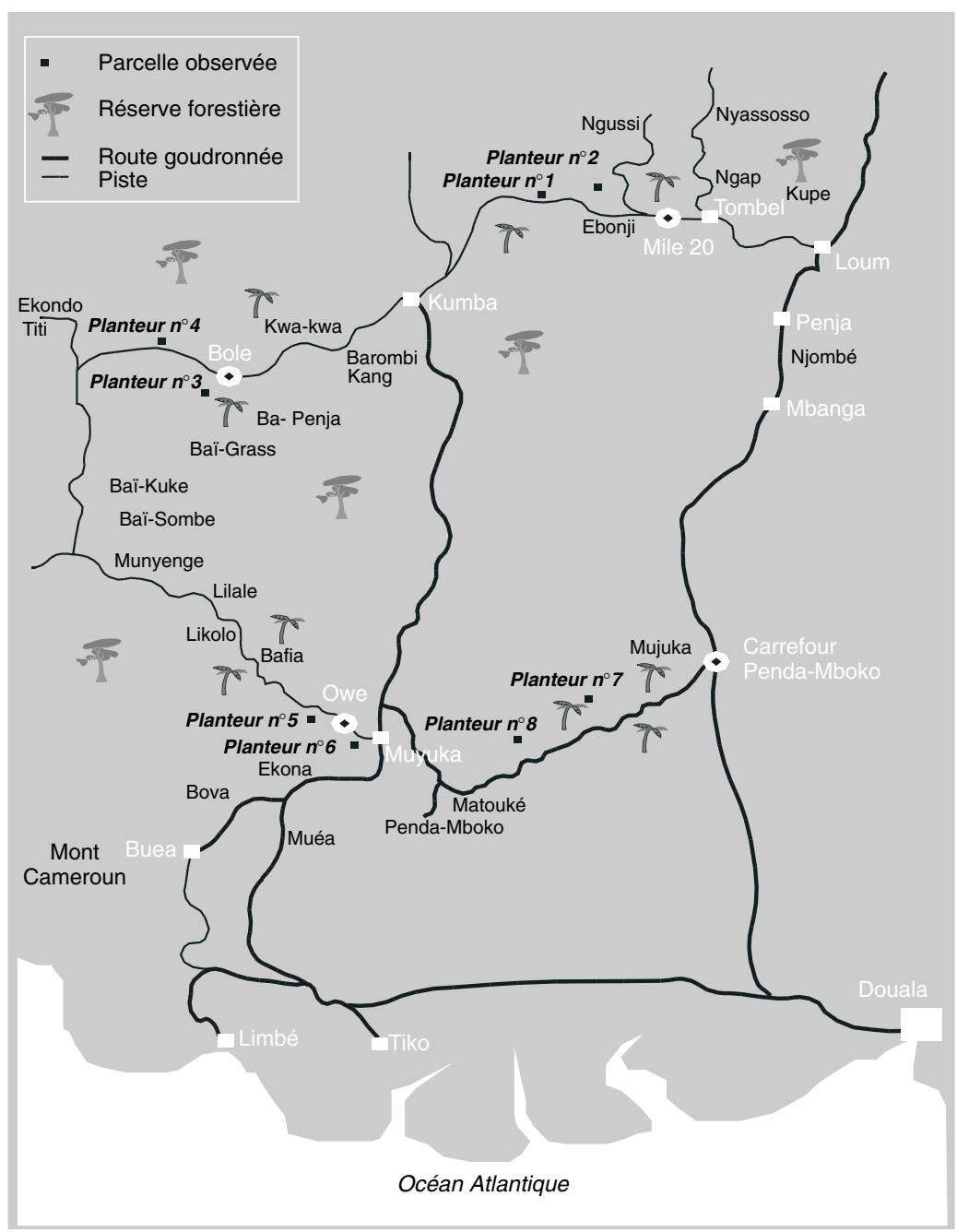

station, en fonction des besoins des producteurs. Pour cela, des diagnostics de filière conduits en 1991 ont permis de localiser les zones de production approvisionnant les grands centres urbains [2, 3]. En prolongement de cette démarche, des enquêtes socio-économiques et des suivis d'exploitation ont permis successivement de mieux connaître les stratégies mises en œuvre [4] et de modéliser le fonctionnement micro-économique des exploitations [5]. Ces travaux ont montré notamment que la production est issue de systèmes associés fortement diversifiés où le bananier plantain est présent de manière très hétérogène. Pour mieux connaître les contraintes qui limitent la production, un " observatoire permanent de la production " a été mis en place dans le cadre d'une démarche interdisciplinaire. Le travail que nous présentons décrit l'approche méthodologique qui a été adoptée par cet observatoire et analyse les résultats obtenus.

\section{Matériel et méthodes}

L'échantillonnage a été raisonné de manière à couvrir les principales zones de production qui participent à l'approvisionnement de Douala, la plus importante ville du Cameroun. Trois zones ont été retenues dans la province du Sud-Ouest et une dans la région de Penda Mboko de la province du Littoral (figure 1). Ces zones représentent des conditions agroécologiques distinctes. Les parcelles choisies appartiennent à des planteurs dont l'objectif de production est de garantir une autosuffisance alimentaire et d'assurer un surplus commercialisable. Les sites ont été choisis en fonction des associations culturales et de la densité de plantation des bananiers plantains. Les données, collectées en milieu paysan, ont été obtenues à partir d'un suivi de parcelles et d'enquêtes effectuées à l'échelle des exploitations pendant deux années : 1997 et 1998.

\subsection{Suivi des parcelles}

Dans chaque parcelle, les observations ont été réalisées sur deux variétés. La première, de type 'French moyen', est commune à 
l'ensemble des parcelles, la seconde a été constituée par l'autre variété dominante de la parcelle ('Faux corne', 'Bâtard', 'Gros Michel', etc.). Pour chacune des parcelles, les données ont été issues :

- d'un diagnostic parcellaire basé sur des analyses annuelles de sol sur 0 à $30 \mathrm{~cm}$ (méthode Cobalt-Hexamine) [6] et des relevés climatiques de chaque zone ;

- d'un suivi mensuel de la plantation jusqu'à la floraison de 30 bananiers observés sur les paramètres : hauteur, circonférence, rythme d'émission foliaire mensuel (ref), rang de la plus jeune feuille nécrosée (pjfn) par la maladie des raies noires (Mycosphaerella fijiensis), nombre de feuilles vivantes (nfv) ;

- d'un suivi bimestriel de 10 bananiers autres que les 30 plants observés précédemment et analysés sur les paramètres : évaluation des populations de nématodes (Radopholus similis, Helicotylenchus multicinctus, Pratylenchus spp., Meloidogyne spp., Hoplolaimus spp.) selon la technique de Vilardebo et Guérout [7] et détermination de l'indice de nécrose racinaire selon Bridge et Gowen [8] ;

- d'un suivi trimestriel de $10 \%$ de la parcelle, dont les 30 bananiers observés mensuellement jusqu'à la floraison, portant sur une estimation de la population de charançons (Cosmopolites sordidus) par piégeage [9] ;

- d'une observation, à la récolte, des plants ayant produit parmi les 30 bananiers observés mensuellement jusqu'à leur floraison. Les paramètres évalués ont alors été le nombre de feuilles vivantes, l'intervalle entre la floraison et la coupe (ifc), une estimation, par décorticage du bulbe, des dégâts dus aux charançons [10] ; le poids et le nombre de mains et de doigts du régime ; les poids, longueurs et grades (= diamètres) moyens des doigts ; la couleur et la dureté de la peau et de la pulpe ; l'incidence des maladies causées par Trachysphaera fructigena et Pyricularia grisea, deux champignons attaquant les fruits.

\subsection{Enquêtes à l'échelle de l'exploitation}

Des questionnaires remis aux planteurs ont permis d'évaluer les quantités de bananes autoconsommées et commercialisées en fonction des différentes parcelles et d'obtenir des indications sur les itinéraires techniques pratiqués.

\section{Résultats sur la connaissance des facteurs limitants}

\subsection{Déterminants économiques des systèmes de culture}

L'ensemble des résultats obtenus a montré que les planteurs combinaient plusieurs parcelles répondant à des objectifs économiques différents, ce que démontre le rapport entre les taux de commercialisation et d'autoconsommation. Trois principaux systèmes de culture ont été identifiés.

- Le bananier plantain peut être exploité en monoculture (figure $2 a$ ). Un tel type de culture, mené en alternance avec des
Figure 2.

Principaux systèmes de culture du plantain identifiés dans le sud-ouest du Cameroun à la suite de deux années d'enquêtes :

(a) plantain exploité en monoculture ;

(b) plantain en association avec des cultures vivrières ; (c) plantain en association de cultures vivrières et de cultures de rente.

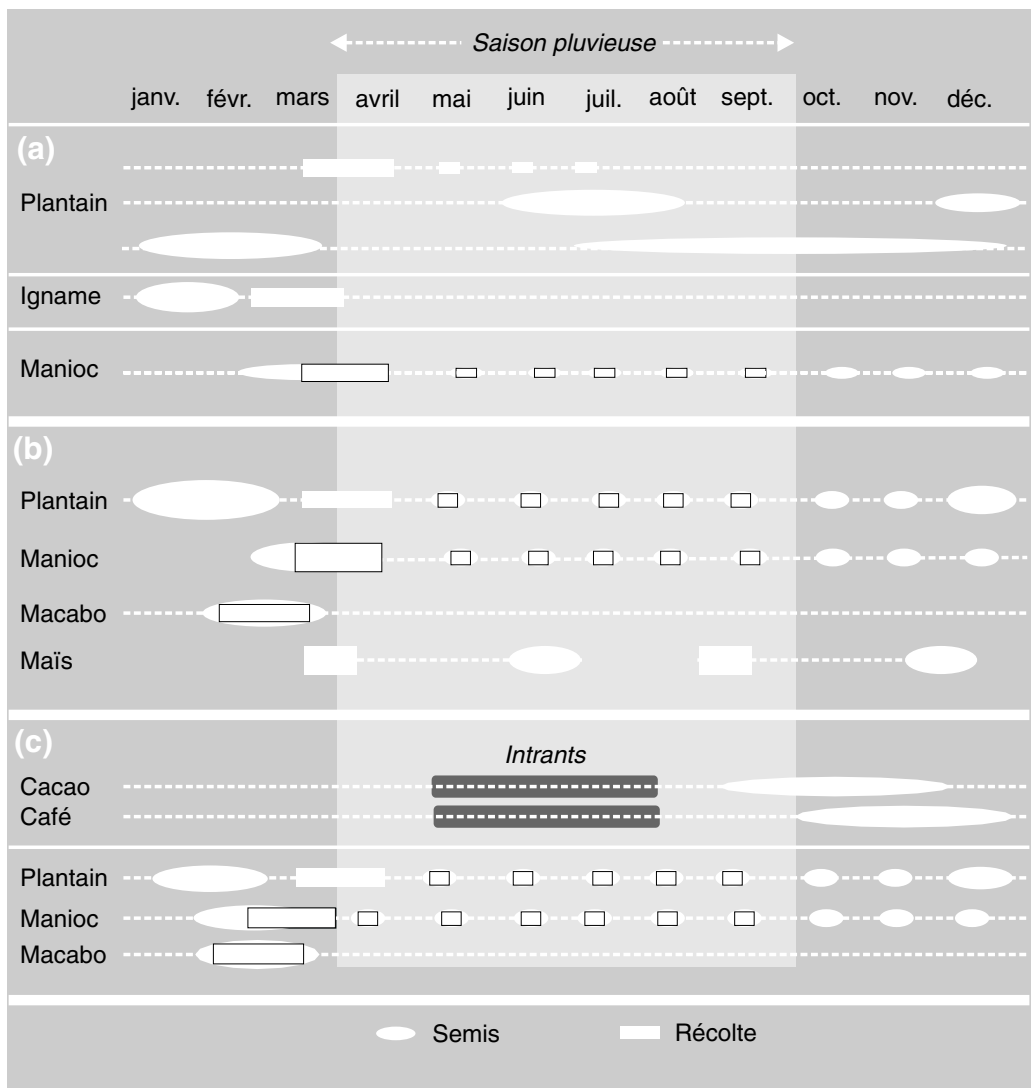


${ }^{1}$ La tontine correspond à une association de personnes versant de l'argent dans une caisse commune dont le montant est remis, à tour de rôle, à chacun de ses membres. jachères courtes de 2 ans sur des sols pauvres, a été observé chez deux planteurs parmi les huit enquêtés. Ces planteurs qui réalisaient une association igname-plantain sur défriche de forêt s'orientent dorénavant vers des monocultures en plantain ou en igname et manioc en alternance avec des périodes de jachères courtes de 2 ans. Dans ce système de culture, les densités de plantation en bananiers plantains sont importantes; les désherbages manuels ont lieu trois fois par an mais aucun intrant chimique n'est utilisé. Ces producteurs ciblent l'obtention de deux revenus principaux : l'un en milieu de saison des pluies correspondant au premier cycle du plantain, l'autre en fin de saison sèche, lors des récoltes d'ignames et de plantains de second cycle. Cette conduite permet également de disposer d'un revenu régulier du fait de l'étalement des récoltes de manioc et des deuxième et troisième cycles de plantain.

- Une association de bananiers plantains et de cultures vivrières (figure $2 b$ ) a été observée chez trois planteurs sur les huit enquêtés. Ce système de culture répond à deux objectifs : celui d'une production essentiellement autoconsommée, ce qui a été observé chez deux planteurs, ou, en plus de ce rôle d'autoconsommation, une commercialisation importante de la production de bananes plantains. Dans ce cas, une corrélation positive entre l'augmentation du taux de commercialisation et la densité de bananiers plantains est observée.
- Une association de cultures vivrières et de cultures de rente (figure 2c) a été le fait de trois des planteurs suivis. Ce système de culture se caractérise par une faible présence des bananiers et autres vivriers. L'objectif principal est de générer un revenu au travers de productions de rentes (café, cacao), de fournir une production vivrière permettant de nourrir la famille et de disposer d'un revenu complémentaire pour l'épargne dans des tontines ${ }^{1}$. Ce mode d'épargne permet de subvenir à des dépenses ponctuelles (santé, éducation, équipement, etc.), mais, surtout, aux besoins saisonniers de trésorerie occasionnés par l'entretien et les traitements phytosanitaires des cultures de café et de cacao.

\subsection{Observations agronomiques}

La fertilité des sols s'est révélée très contrastée (tableau I) sur des sols ferralitiques jaunes, très pauvres, présents chez trois planteurs et des sols ferralitiques jeunes et sols volcaniques très riches chez les autres. Cette diversité est conforme aux caractéristiques pédologiques de cette zone. Le climat est de type tropical humide ; il se caractérise par une température uniforme toute l'année ( $26^{\circ} \mathrm{C}$ en moyenne) et par l'alternance de deux saisons : une saison sèche de novembre à mars, suivie d'une saison des pluies. Les précipitations moyennes annuelles s'échelonnent entre (2000 et 4000) $\mathrm{mm}$ par an. Elles atteignent leur régime maximal entre juillet et août.

Tableau I.

Composition des sols dans huit bananeraies suivies quant à leur production dans le sud-ouest du Cameroun.

\begin{tabular}{|c|c|c|c|c|c|c|c|c|}
\hline Élément & $\begin{array}{c}\text { Plantation } 1 \\
\text { Ferralitique jeune }\end{array}$ & $\begin{array}{l}\text { Plantation } 2 \\
\text { e Volcanique }\end{array}$ & $\begin{array}{c}\text { Plantation } 3 \\
\text { Ferralitique jeune }\end{array}$ & $\begin{array}{c}\text { Plantation } 4 \\
\text { Ferralitique jaune }\end{array}$ & $\begin{array}{c}\text { Plantation } 5 \\
\text { Volcanique jeune }\end{array}$ & $\begin{array}{c}\text { Plantation } 6 \\
\text { Volcanique jeune }\end{array}$ & $\begin{array}{c}\text { Plantation } 7 \\
\text { Ferralitique jaune }\end{array}$ & $\begin{array}{c}\text { Plantation } 8 \\
\text { Ferralitique jaune }\end{array}$ \\
\hline Matière organique (\%) & 5,3 & 12 & 4,2 & 1,8 & 20 & 17,2 & 1,36 & 1,4 \\
\hline $\mathrm{Ca}\left(\mathrm{mEq} \cdot 100 \mathrm{~g}^{-1}\right)$ & 4,6 & 22 & 13,7 & 1,7 & 35,6 & 33,8 & 0,9 & 1,8 \\
\hline $\mathrm{Mg}\left(\mathrm{mEq} \cdot 100 \mathrm{~g}^{-1}\right)$ & 1,9 & 5 & 4,3 & 0,5 & 4,8 & 5,4 & 0,3 & 0,5 \\
\hline $\mathrm{K}\left(\mathrm{mEq} \cdot 100 \mathrm{~g}^{-1}\right)$ & 0,9 & 0,6 & 0,5 & 0,15 & 0,66 & 1 & 0,06 & 0,20 \\
\hline S/CEC (\%) & 66 & 90 & 91 & 54 & 97 & 98 & 78 & 83 \\
\hline $\mathrm{pH}$ & 5,75 & 6,5 & 6,1 & 5,0 & 7,3 & 7,4 & 5,4 & 5,3 \\
\hline
\end{tabular}

S/CEC = somme des cations échangeables / capacité d'échange cationique. 
Il est à noter que les producteurs qui ont été enquêtés n'appliquaient ni fertilisation minérale, ni pesticides dans leurs parcelles de bananiers.

\subsubsection{Observations des bananiers en phase végétative}

\subsubsection{Rythme d'émission foliaire}

Quelle que soit la parcelle suivie, l'émission foliaire des bananiers a été très faible en fin de saison sèche : moins de deux feuilles émises par mois (figure 3). En mars, avec la reprise des pluies, l'émission foliaire a augmenté très rapidement pour atteindre un rythme de trois ou quatre feuilles par mois. D'août à septembre, l'émission foliaire a diminué pour atteindre deux ou trois feuilles par mois, alors que, entre octobre et décembre, il y a eu de nouveau une légère augmentation : trois ou quatre feuilles émises chaque mois. À partir du mois de janvier (saison sèche), l'émission foliaire est redevenu faible.

Ces variations du rythme d'émission foliaire ne peuvent pas être expliquées par la seule pluviométrie. Ainsi, d'une zone à l'autre, alors que la pluviométrie était homogène, l'émission foliaire a montré des écarts. Parmi les facteurs pouvant interférer, on peut évoquer la conduite en touffes pratiquée chez l'un des planteurs, la composition chimique des sols moins riche chez un autre planteur ou des densités de plantation différentes entre certaines parcelles, celles, en particulier, des septième et huitième planteurs enquêtés dont la densité des plants était de (650 et 1050$)$ pieds par ha, respectivement.

\subsubsection{Nombre de feuilles vivantes et rang de la plus jeune feuille nécrosée}

L'évolution au cours de l'année du nombre de feuilles vivantes a été plus ou moins identique à celle du rythme d'émission foliaire, à l'exception, cependant, de la période d'octobre à décembre.

Ce caractère a pourtant été plus variable d'une parcelle à l'autre. Ainsi, chez deux planteurs sur huit, l'augmentation du nombre de feuilles vivantes observée en mars-avril a été d'une seule feuille, alors
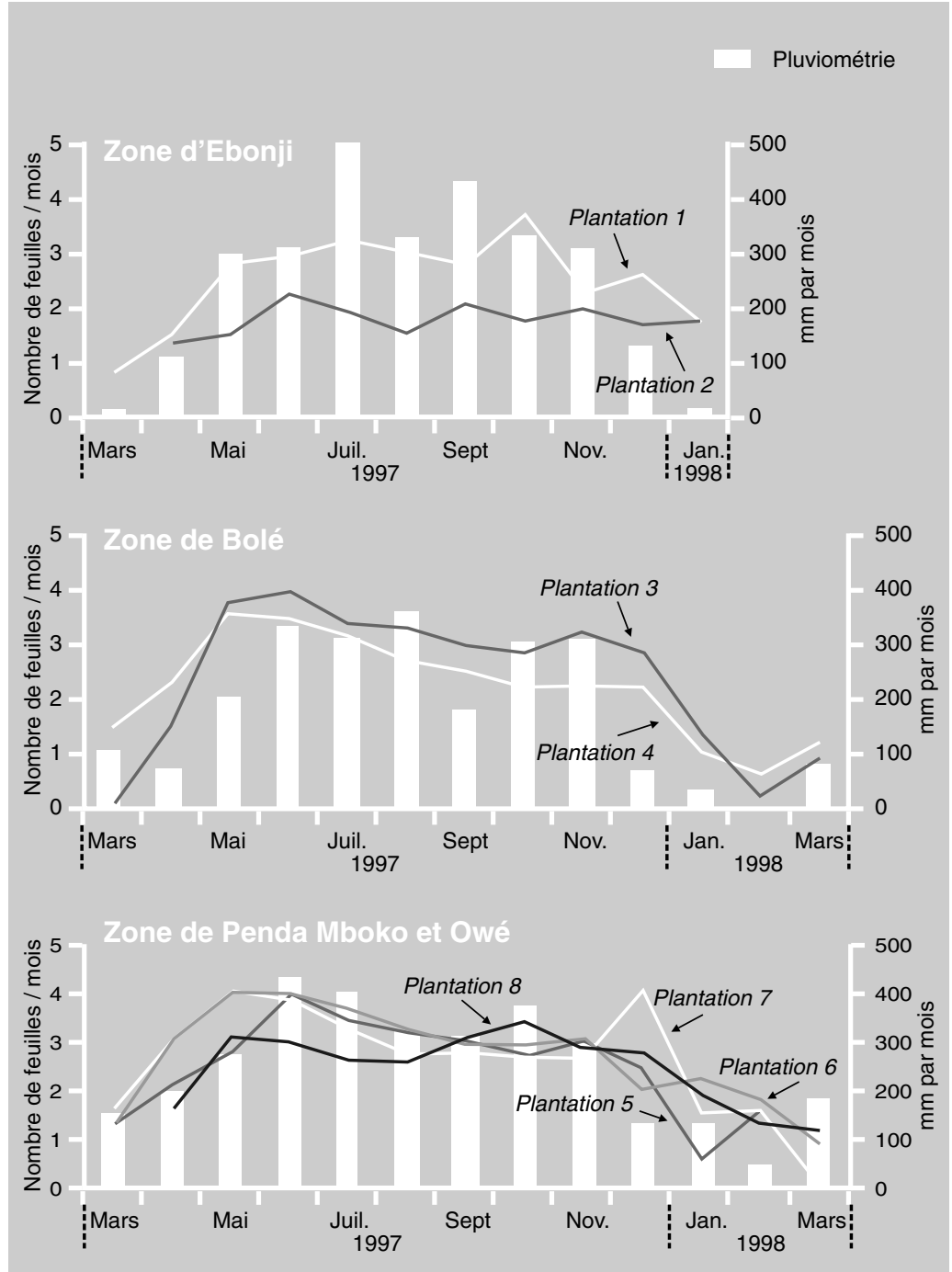

que chez les six autres, elle était de trois à trois feuilles et demi. Par ailleurs, chez deux planteurs, le nombre de feuilles vivantes par bananier a été maintenu au-dessus de cinq en fin de saison sèche.

Le rang de la plus jeune feuille nécrosée est resté relativement stable pendant toute la période d'observation se situant à la valeur de 3,5 sur deux parcelles et à la valeur de 4,5 sur les autres. Ces faibles valeurs, similaires à celles obtenues par différents auteurs au Gabon [11], Zaïre [12] et Cameroun [13] reflèteraient une grande sensibilité des plantains à la maladie de la cercosporiose noire.

\section{Figure 3.}

Relation entre le rythme d'émission foliaire mensuel de bananiers plantains et la pluviométrie dans différentes zones au sud-ouest du Cameroun. 


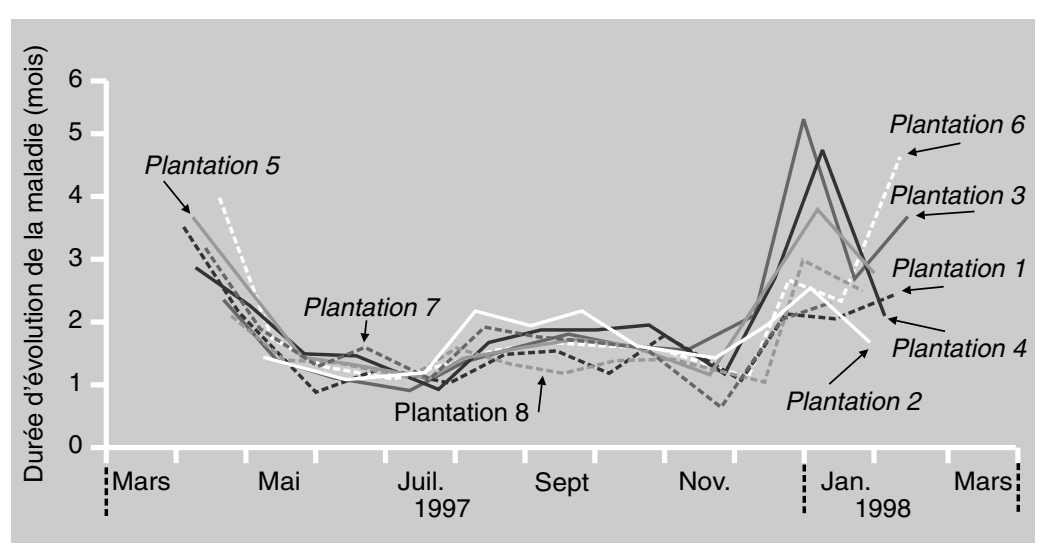

Figure 4.

Évolution de la durée entre la date d'émission de la feuille et l'apparition des nécroses (dem) sur l'ensemble de huit parcelles suivies entre 1997 et 1998 dans le sud-ouest du Cameroun. $($ dem $)=[($ rang de la plus jeune feuille nécrosée) / (rythme d'émission foliaire mensuel)].
Figure 5.

Relation entre la circonférence du pseudotronc à la floraison et la somme des cations échangeables dans le sol pour huit bananeraies suivies entre 1997 et 1998 dans le sud-ouest du Cameroun.
Le rapport [(rang de la plus jeune feuille nécrosée)/ (rythme d'émission foliaire mensuel)] permet d'estimer la durée d'évolution totale de la maladie (dem) : durée entre la date d'émission de la feuille et l'apparition des nécroses. L'évolution de la (dem) au cours du temps a été similaire sur toutes les parcelles (figure 4). Elle semble évoluer selon un caractère saisonnier atteignant ses plus fortes valeurs en saison sèche. Cette évolution lente de la maladie en saison sèche est en accord avec les résultats obtenus au Nigeria [14].

\subsubsection{Observations des bananiers à la floraison}

\subsubsection{Circonférence}

La circonférence des bananiers à la floraison a varié en moyenne de $(70$ à 56$) \mathrm{cm}$ entre les bananiers ayant fleuris en janvier et ceux ayant fleuris en juillet. À partir d'août, la circonférence a augmenté pour

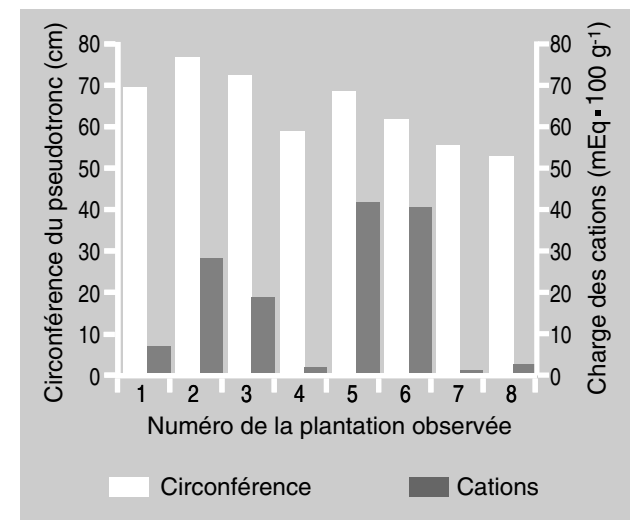

atteindre $67 \mathrm{~cm}$ en moyenne au mois de décembre. Ces variations de croissance sont à rapprocher des variations saisonnières du rythme d'émission foliaire, ce qui renvoie aux effets du climat, de la fertilité et des techniques culturales. Sur trois parcelles, la circonférence moyenne annuelle à la floraison a été inférieure à $58 \mathrm{~cm}$, alors qu'elle a été supérieure à $67 \mathrm{~cm}$ pour les bananiers de quatre autres parcelles. Chez l'un des planteurs, l'observation de circonférences de faible taille pourrait être expliquée par la pratique d'un effeuillage excessif effectué sur le pseudotronc.

La circonférence à la floraison apparaît liée aux types de sols et à leur composition chimique (figure 5). Sur les sols volcaniques et ferralitiques jeunes, peu désaturés, la croissance des bananiers a été très bonne. Les sols ferralitiques jaunes très pauvres ont donné des résultats médiocres. La circonférence du pseudotronc apparaît être un bon descripteur global de la croissance du bananier pendant la phase végétative car elle intègre, durant cette période, les effets des techniques culturales, du climat et de la fertilité du sol.

\subsubsection{Nombre de doigts}

Le nombre moyen de doigts à la date de floraison a diminué de 70 à 52 entre janvier et juillet. Il a augmenté ensuite pour atteindre 66 doigts en janvier. Il existe une forte disparité d'une parcelle à l'autre : quatre d'entre elles ont permis d'obtenir des régimes ayant, en moyenne, moins de 55 doigts, alors que, chez les quatre autres parcelles, les régimes en ont présenté plus de 65. Ces différences sont à imputer essentiellement à la croissance, car il y a eu une bonne corrélation entre la circonférence des bananiers à la floraison et le nombre de doigts $(r=0,72)$ (figure 6). Ces résultats sont conformes à certains autres déjà obtenus sur bananiers dessert [15]. Le nombre de doigts se révèle minimal lors de la saison des pluies, ce qui pourrait traduire l'impact du stress de la saison sèche sur l'initiation florale.

\subsubsection{Nombre de feuilles vivantes et rang de la plus jeune feuille nécrosée}

Le nombre de feuilles vivantes à la floraison a augmenté de 6 à 10 feuilles entre 
janvier et juillet, puis il a diminué jusqu'à 6,5 feuilles en décembre. Comme pour la phase végétative, cette évolution peut être reliée à celles des rythmes d'émission foliaire et des vitesses d'évolution de la maladie des raies noires durant les mois précédents.

Le rang de la plus jeune feuille nécrosée à la floraison a varié, suivant les parcelles, entre 2 et 4, ce qui est très faible. De tels résultats pourraient être expliqués par la sensibilité des bananiers à la maladie de la cercosporiose noire et l'absence de traitement fongicide dans la zone.

\subsubsection{Observations des bananiers à la récolte}

Le poids d'un doigt est très lié à son grade et à sa longueur $(r=0,80)$ (figure 7$)$. Par ailleurs, le grade et la longueur sont très corrélés $(r=0,95)$, sauf en saison sèche où le décrochement de la longueur observé pourrait être lié à un stress hydrique. Ces composantes ont varié en fonction des parcelles. Pour trois d'entre elles, le poids moyen d'un doigt a été inférieur à $137 \mathrm{~g}$ alors qu'il a été supérieur à $170 \mathrm{~g}$ chez les autres. Les paramètres poids, grade et longueur d'un doigt se sont révélés très liés au poids du régime (figures 7,8 ) qui a luimême varié de (6 à 8) kg pour les bananiers de trois des huit parcelles suivies et de (9 à 13) kg pour les cinq autres. Le poids très faible des régimes de bananiers du premier groupe de parcelles pourrait être expliqué par la faible fertilité des sols (ferralitique jaune). Dans le cas du deuxième groupe où les sols sont plus riches en cations échangeables (sols volcaniques et ferralitiques jeunes), il est apparu que les planteurs récoltaient précocement pour se protéger des vols de régimes. L'intervalle de temps écoulé entre la floraison et la coupe du régime a été en effet inférieur à 70 j, c'est-à-dire en-dessous du stade de récolte considéré optimal pour favoriser le remplissage des doigts.

\subsection{Importance du parasitisme tellurique}

Les principales espèces de nématodes rencontrées ont été Radopholus similis,

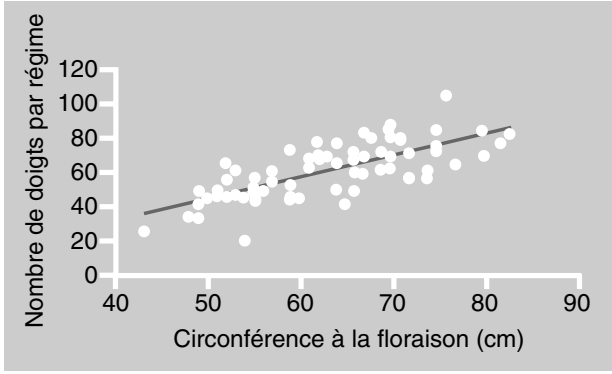

Figure 6.

Relation entre la circonférence du pseudotronc à la floraison et le nombre de doigts portés par le régime récolté.

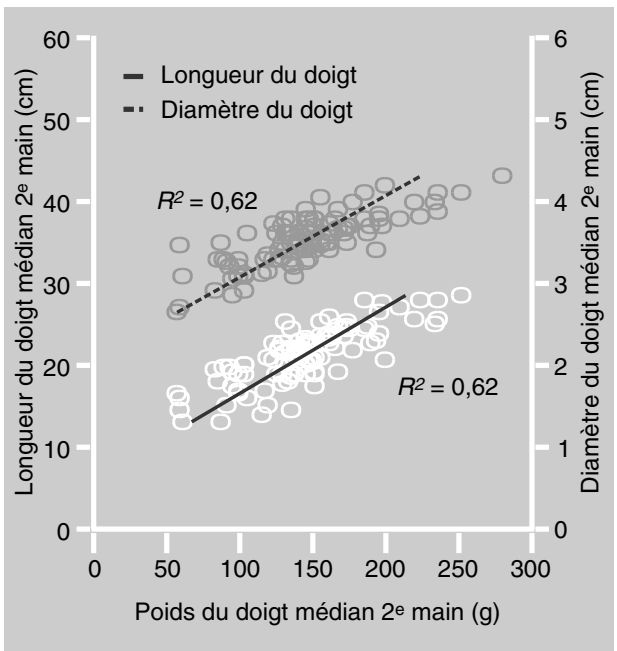

Figure 7.

Relation entre les valeurs moyennes du poids d'un doigt de plantain et les paramètres longueur et diamètre (= grade) de ce doigt. Résultats de 30 bananiers observés pour chacune de huit parcelles suivies entre 1997 et 1998 dans le sudouest du Cameroun.

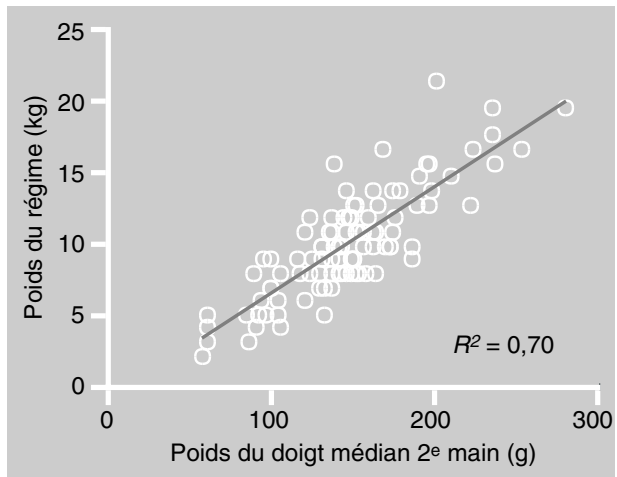

Figure 8.

Relation entre les valeurs moyennes du poids d'un doigt et le poids du régime. Résultats de 30 bananiers observés pour chacune de huit parcelles suivies entre 1997 et 1998 dans le sud-ouest du Cameroun.
Pratylenchus spp., Helicotylenchus multicinctus, Meloidogyne spp. et Hoplolaimus spp.

L'importance des espèces a varié selon les parcelles. $R$. similis a été l'espèce principale sur deux d'entre elles chez lesquelles l'espèce a été présente à un taux supérieur au seuil de dégâts qui est estimé à 10000 individus de $R$. similis par $100 \mathrm{~g}$ de racines. Ce 
Figure 9.

Répartition et effectifs des populations de nématodes rencontrées, entre 1997 et 1998 , par suivi de huit parcelles de bananiers plantains dans le sud-ouest du Cameroun.

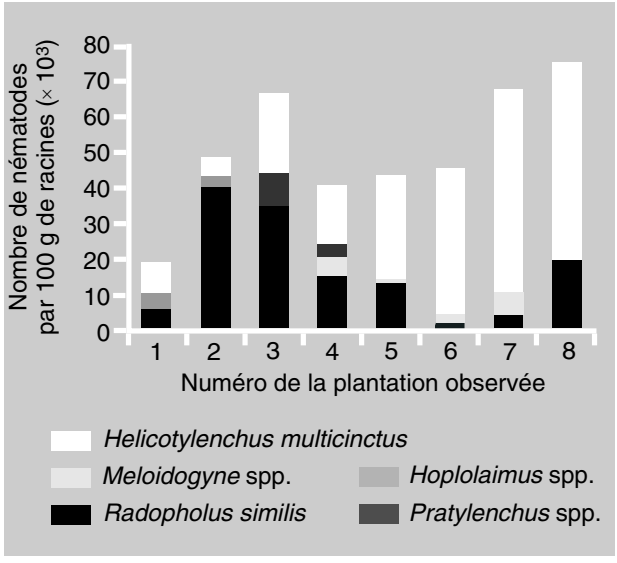

Figure 10.

Taux de pertes en bananiers, évalué par parcelle, dans huit plantations suivies entre 1997 et 1998 dans le sud-ouest du Cameroun.

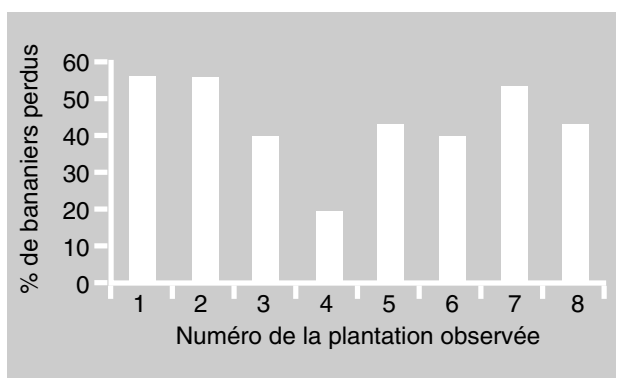

nématode est un endoparasite migrateur bien connu sur bananier qui occasionne, sur les racines, des lésions rougeâtres profondes [16]. Sur les six autres parcelles, l'espèce de nématodes dominante a été H. multicinctus (figure 9). Par ailleurs, Meloidogyne spp. et Hoplolaimus spp. ont été observés sur la quasi-totalité des plantations, mais leur niveau de population est resté faible.

La dynamique de développement des populations de nématodes semble avoir été identique sur l'ensemble des parcelles. Les pics de population ont été obtenus entre juillet et septembre, ce qui coïncide avec les périodes de forte pluviométrie.

Sur quatre parcelles, les racines, dont l'indice de nécrose racinaire (inr) a varié de (0 à 25) \%, ont présenté un état sanitaire satisfaisant, alors que, sur les quatre autres, des (inr) plus élevés, car variant de (25 à 50) \%, ont témoigné d'un plus fort impact du parasitisme tellurique. Sur les parcelles où H. multicinctus était l'espèce dominante, les dégâts racinaires ont généralement été faibles, alors que les plus fortes attaques ont été rencontrées sur les parcelles où $R$. similis dominait. Une forte corrélation $(r=0,92)$ a été mise en évidence entre le niveau moyen par parcelle des populations de $R$. similis et l'indice de nécrose racinaire moyen par parcelle.

Le charançon Cosmopolites sordidus a été rencontré sur l'ensemble des parcelles. Les niveaux d'infestation ont été plus ou moins importants. La capture des adultes a permis de dénombrer entre (0 et 3,1) charançons par piège. Les dégâts occasionnés ont été relativement faibles et ils ont été fortement variables au sein de chacune des parcelles. Nous avons pu observer une bonne relation entre les résultats obtenus lors du piégeage des adultes de charançons et ceux relevés après décorticage des bulbes : à l'exception des bananiers d'une seule parcelle, les plants situés sur les parcelles les plus infestées ont présenté des bulbes à forts coefficients d'infestation et inversement.

\subsection{Pertes avant récolte}

Parmi les bananiers plantains qui ont été suivis, $40 \%$ en moyenne ne sont pas parvenus à une maturité suffisante pour pouvoir être consommés. Ces pertes au champ ont été dues tout d'abord à des bananiers dont le pseudotronc a été cassé en son milieu sous l'influence du stress hydrique survenu en fin de saison sèche (mois de mars). Ensuite, en avril-mai, malgré la reprise des pluies, les pertes sont restées importantes, du fait surtout de bananiers déracinés. Après ces périodes, les pertes ont diminué et leur taux est resté stable tout au long de l'année. Elles ont été alors uniquement occasionnées par la chute de bananiers ou leur dépérissement en cours de développement. Le vol a pu atteindre $20 \%$ de la production.

Le taux de pertes a été relativement semblable d'une parcelle à l'autre (figure 10), alors que les causes de ces pertes ont varié. Il y eu davantage de pseudotroncs cassés sur les sols disposant d'une faible capacité de rétention en eau (figure 11). Par ailleurs, le nombre de bananiers déracinés par 
parcelle s'est révélé très lié à l'état sanitaire des racines, ce qui est illustré par la forte corrélation $(r=0,74)$ trouvée entre le pourcentage de chutes par parcelle et la présence du nématode $R$. similis. Cependant, l'une des parcelles dont les bananiers présentaient un fort (inr) n'a eu aucune perte à imputer à la chute de plants du fait d'un tuteurage systématique effectué par le planteur concerné.

\subsection{Maladies des fruits}

Deux maladies ont affecté la production des bananiers étudiés, dépréciant leur qualité : la maladie du bout du cigare causée par Trachysphaera fructigena et une maladie causée par Pyricularia grisea. Ces maladies n'ont été rencontrées que sur deux zones, dans quatre parcelles. Le pourcentage de régimes attaqués est cependant resté faible ; il a atteint un maximum de $15 \%$ par planteur, principalement en saison des pluies, le reste de l'année il est resté très négligeable.

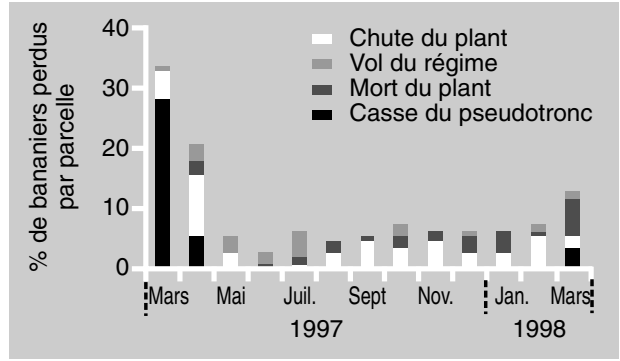

\section{Interprétation des résultats}

Le suivi des exploitations a mis en évidence les faibles ressources financières des producteurs qui pratiquent par conséquent une culture sans intrants. De fait, lorsque les rendements en bananes plantains diminuent après le deuxième cycle, le bananier est remplacé par le manioc dans le système de culture.

L'identification et la hiérarchisation des facteurs qui déterminent le rendement en milieu paysan montre que ce dernier dépend fortement du pourcentage de plants

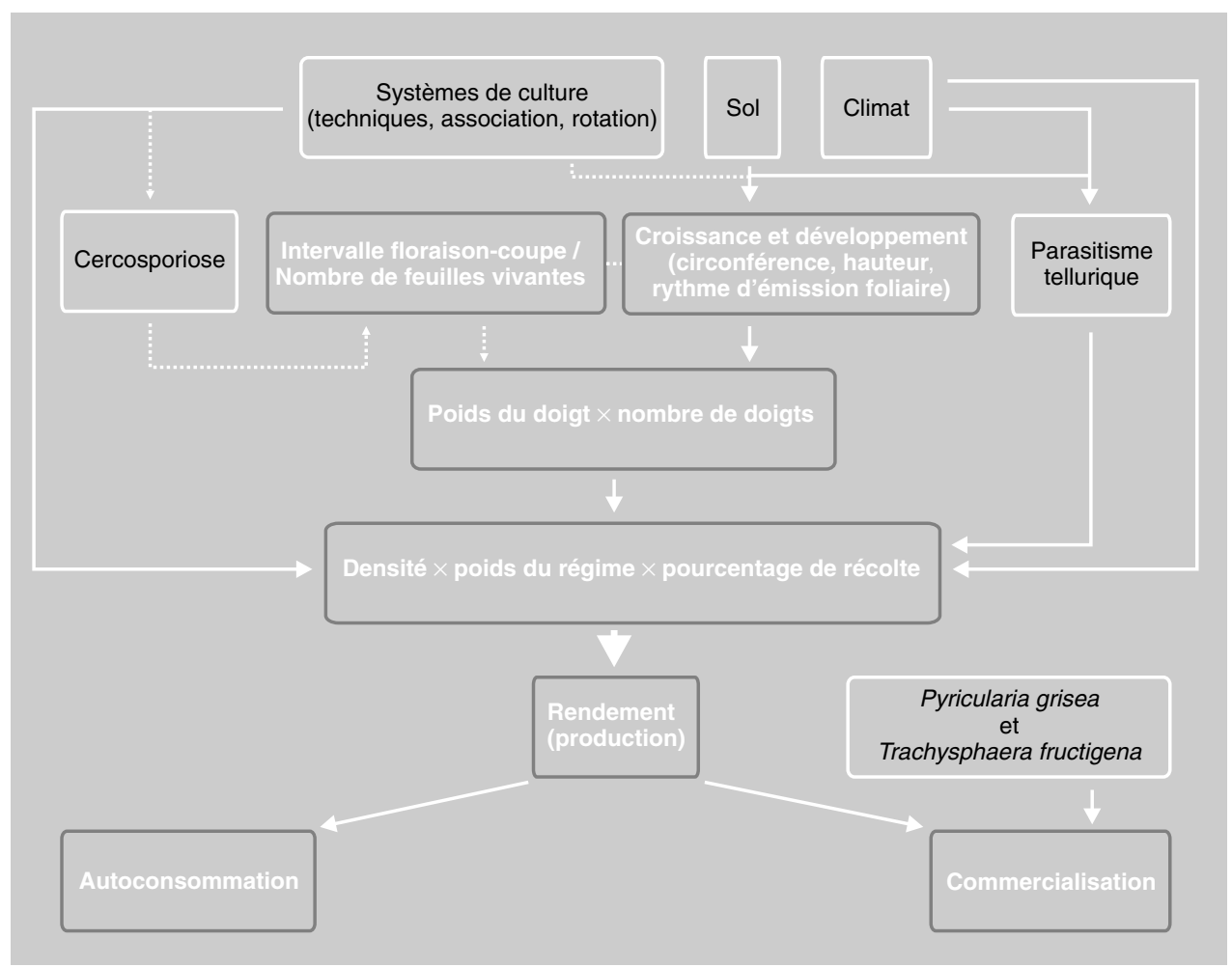

Figure 12.

Schéma récapitulatif des facteurs déterminant la production de bananiers plantains d'après des résultats issus du fonctionnement d'une cellule d'observation dans le sud-ouest du

Cameroun. pertes en bananiers au cours de l'année et origine de ces pertes, par suivi de huit du Cameroun. 
récoltés (figure 12). Sur une parcelle donnée, plus de $30 \%$ des bananiers, en moyenne, ne parviennent pas à produire un régime ayant une maturité suffisante pour pouvoir être consommés. Ces pertes s'expliquent à $50 \%$ par des bananiers qui se déracinent, cette situation étant principalement liée à la présence du nématode $R$. similis. Les dégâts occasionnés par ce ravageur sur les racines fragilisent le système racinaire et augmentent la sensibilité des bananiers à la chute. La pratique du tuteurage observé sur l'une des bananeraies suivies semble avoir fortement limité l'impact de ce parasitisme tellurique. Bien que nos résultats aient permis de noter la présence du charançon noir sur toutes les parcelles observées, les niveaux d'attaque n'ont pas été très élevés. Près de $40 \%$ des pertes ont pu être expliqués également par l'impact de la saison sèche qui provoque une baisse de la pression des liquides contenus dans le bananier et favorise la casse du bananier au niveau du pseudotronc. Ces pertes touchent exclusivement les bananiers ayant fleuri ou proches de la floraison et la répercussion sur l'offre se fait entre mai-juillet, puisque c'est à cette période que la récolte aurait dû avoir lieu. Sur les marchés urbains, le plantain devient alors un produit de luxe pour de nombreux citadins qui préfèrent se rabattre vers des produits d'importation moins chers ou vers l'achat de manioc.

Le rendement dépend aussi du poids du régime et donc de son nombre de doigts et de leur remplissage. Le nombre de doigts s'est révélé très lié au développement végétatif du bananier et, plus particulièrement, à la circonférence du pseudotronc à la floraison, ce qui représente un indicateur facilement mobilisable pour des diagnostics rapides. Pour le remplissage du doigt, le protocole du suivi des parcelles n'a pas pu permettre de montrer l'incidence de la maladie des raies noires sur les composantes du rendement. Précédemment, Stover [17] et Mobambo [18], respectivement au Honduras et au Nigéria, avaient mis en évidence des réductions de rendement de (50 et 33) \% dues à cette maladie. Leurs études comparaient des parcelles traitées ou non traitées aux fongicides et considéraient un paramètre de sévérité de la maladie tel que le pourcentage de surface foliaire malade. L'utilisation de ce dernier paramètre, ainsi que la réalisation d'analyses statistiques plus poussées à partir de données sur une plus longue période, devrait permettre de mieux dégager l'incidence de ce facteur et de situer son impact par rapport aux autres contraintes. En l'état actuel de nos travaux et en l'absence d'une comparaison avec des parcelles de références traitées, il apparaît difficile d'obtenir une évaluation absolue de l'impact de cette maladie.

\section{Conclusion et perspectives}

L'analyse du rendement des bananeraies qui ont été suivies est difficile du fait de la complexité des systèmes de culture qui ont été observés dans les conditions de production étudiées. Pour améliorer le dispositif d'étude, il serait nécessaire d'affiner le protocole de suivi.

En raison des forts taux de perte avant récolte à certaines périodes, très peu de bananiers ont été récoltés, ce qui rend l'interprétation des données entre la floraison et la récolte très délicate. De ce fait, davantage de bananiers, par exemple 50 au lieu de 30, devraient être suivis dans chacune des parcelles soumises à l'enquête. De même, si l'impact sur le rendement des parasites et ravageurs est apparu élevé, la dynamique d'évolution dans le temps de ces attaques n'a pu être appréhendée. La réalisation des piégeages de charançons, et notamment le nombre de pièges et la fréquence des observations, devrait être améliorée. Par ailleurs, le rôle de la fertilité sur le rendement n'a pas été clairement caractérisé. Enfin, les observations concernant la cercosporiose ont montré de très fortes infestations sur une partie de l'année. Cependant, les différences étant essentiellement saisonnières et le remplissage du régime dépendant aussi de nombreux autres paramètres, il a été difficile de quantifier l'impact de cette maladie. L'observation d'un paramètre de sévérité de la maladie des raies noires permettrait d'évaluer plus précisément cette contrainte. Pour 
l'ensemble de ces paramètres, un nombre supérieur de parcelles suivies serait nécessaire.

Le niveau d'observation privilégié étant celui de la parcelle, un meilleur suivi des paramètres économiques à l'échelle de l'exploitation apparaît nécessaire pour mieux prendre en compte les déterminants socioéconomiques des rendements et mettre au point un conseil de gestion adapté aux contraintes de financement des petits producteurs de bananes plantains au Cameroun.

\section{Références}

[1] Temple L., Engola Oyep, La sécurité alimentaire en Afrique centrale, in: Enjeux pour un troisième millénaire, Afrique agriculture 279 (2000) 24-26.

[2] Lendres P., Analyse de la filière de commercialisation du plantain au Cameroun, ESAT, CNEARC, mémoire d'ingénieur en agronomie tropicale, Montpellier, France, 1990 $150 \mathrm{p}$.

[3] Temple L., Chataigner J., Kamajou F., Le marché du plantain au Cameroun, des dynamiques de l'offre au fonctionnement du système de commercialisation, Fruits 51 (2) (1996) 83-98.

[4] Temple L., Genettais T., Ganry J., Chataigner J., Les systèmes de production du plantain et les perspectives d'intensification dans le sud-ouest du Cameroun, Fruits 48 (1993) 119-123.

[5] Temple L., Fadani A., Cultures d'exportation et cultures vivrières au Cameroun, éclairage d'une controverse par une modélisation micro-économique, Écon. Rural. 239 (1997) 40-48.

[6] Ciesielsky H., Sterckeman T., Determination of cations exchange capacity and exchangeable cations in soils by means of cobalthexamine trichoride, Effects of experimental conditions, Agronomie 17 (1991) 1-7.

[7] Vilardebo A., Guérout A., Technique d'extraction de Radopholus similis des racines de bananiers, Phytiatr. Phytopharm. 149 (1974) 24-29.
[8] Bridge J., Gowen S., Visual assessment of plant-parasitic nematode and weevil damage on bananas and plantains, in: Gold C.S., Gemmill B. (Eds.), Proc. Res. Coord. Meet., Cotonou, Bénin, 1993.

[9] Delattre P., Recherche d'une méthode d'estimation des populations du charançon du bananier Cosmopolites sordidus Germ. (Coleoptera - Curculionidae), Acta Oecol.Oec. Appl. 1 (1980) 83-92.

[10] Vilardebo A., Le coefficient d'infestation, critère d'évaluation du degré d'attaques des bananeraies par Cosmopolites sordidus Germ., le charançon noir du bananier, Fruits 28 (6) (1973) 417-426.

[11] Fouré É., Les cercosporioses du bananier et leurs traitements : comportement des variétés, étude de la sensibilité variétale des bananiers et plantains à Mycosphaerella fijiensis Morelet au Gabon, Fruits 37 (12) (1982) 749-766.

[12] Mobambo K.N., Naku M., Nganga Z., Black leaf streak disease in banana and plantain in Zaïre, Infomusa 2 (1993) 14-15.

[13] Fouré É., Moulion-Péfoua A., Mourichon X., Étude de la sensibilité variétale des bananiers et des plantains à Mycosphaerella fijiensis Morelet au Cameroun. Caractérisation de la résistance au champ des bananiers appartenant à divers groupes génétiques, Fruits 45 (4) (1990) 339-345.

[14] Mobambo K.N., Gauhl F., Pasberg-Gauhl C., Zuofa K., Season and plant age effect evaluation of plantain for response to black sigatoka disease, Crop Prot. 15 (1996) 609-614.

[15] Dorel M., Perrier X., Influence du milieu et des techniques culturales sur la productivité des bananiers de Guadeloupe, enquêtediagnostic, Fruits 45 (3) (1990) 237-244.

[16] Fogain R., Effect of Radopholus similis on plant growth and yield of plantains (Musa, AAB), Nematology 2 (2000) 129-133.

[17] Blake C.D., Nematode parasites of bananas and their control, in: Peachey J.E. (Ed.), Nematodes of tropical crops, Tech. Commonw. Bur. Helminth. 40 (1969) 109-132.

[18] Stover R.H., Effet du cercospora noir sur les plantains en Amérique centrale, Fruits 38 (4) (1983) 326-329. 


\section{Determinantes de la producción de plátano en el sudoeste de Camerún: interés de un observatorio de la producción.}

Resumen - Introducción. El plátano es un cultivo importante para la seguridad alimentaria en África subsahariana. Para jerarquizar mejor las prioridades de investigación en función de las limitaciones de los productores se ha establecido una célula de observación permanente en el marco de una iniciativa interdisciplinar. Material y métodos. Se efectuó un muestreo de las plantaciones abarcando las zonas de diferentes contextos agroecológicos que abastecen Duala. Se obtuvieron los datos mediante seguimiento de parcelas y explotaciones. A nivel de las parcelas, las observaciones se dirigieron hacia las prácticas de cultivo, poblaciones de nematodos y picudos así como los rendimientos. A nivel de las explotaciones, se efectuó el seguimiento de las funciones económicas del plátano. Resultados y discusiones. El abanico de situaciones culturales estudiadas corresponde a una diversidad de contextos y de objetivos de las explotaciones agrícolas. Las observaciones efectuadas en bananos (ritmo de emisión foliar, diámetro del seudotallo, número de dedos y de hojas vivas, peso y longitud de los dedos, peso del racimo) permitieron explicar cómo se determinaba el rendimiento. Éste se ve sobre todo afectado por las pérdidas precosecha relacionadas con el estado sanitario de las raíces y la presencia de nematodos y de picudos. Tales pérdidas alcanzaron su pico máximo al final de la temporada seca. El rendimiento está también relacionado con el peso del racimo, determinado por el número de dedos que, a su vez, está esencialmente vinculado al crecimiento y llenado de los frutos. Para este último aspecto, los resultados obtenidos no evidencian claramente la incidencia de la Sigatoka negra. Se proponen mejoras para el protocolo de seguimiento.

Musa (platanos) / Camerún / procedimiento de la encuesta / caracteres de rendimiento / enfermedades fungosas / parasitismo

To access this journal online: www.edpsciences.org 